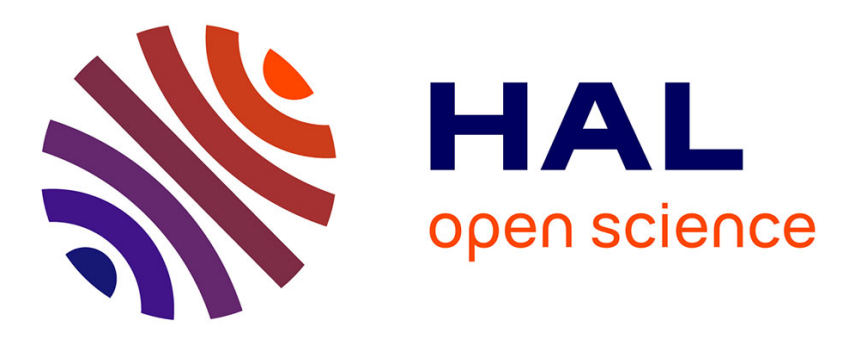

\title{
Influence of roughness on near-field heat transfer between two plates
}

Svend-Age Biehs, Jean-Jacques Greffet

\section{To cite this version:}

Svend-Age Biehs, Jean-Jacques Greffet. Influence of roughness on near-field heat transfer between two plates. Physical Review B: Condensed Matter and Materials Physics (1998-2015), 2010, 82, pp.245410. 10.1103/PhysRevB.82.245410 . hal-00679137

\section{HAL Id: hal-00679137 \\ https://hal-iogs.archives-ouvertes.fr/hal-00679137}

Submitted on 15 Dec 2015

HAL is a multi-disciplinary open access archive for the deposit and dissemination of scientific research documents, whether they are published or not. The documents may come from teaching and research institutions in France or abroad, or from public or private research centers.
L'archive ouverte pluridisciplinaire HAL, est destinée au dépôt et à la diffusion de documents scientifiques de niveau recherche, publiés ou non, émanant des établissements d'enseignement et de recherche français ou étrangers, des laboratoires publics ou privés. 


\title{
Influence of roughness on near-field heat transfer between two plates
}

\author{
S.-A. Biehs and J.-J. Greffet \\ Laboratoire Charles Fabry, Institut d'Optique, CNRS-Université Paris-Sud, \\ Campus Polytechnique, RD128, 91127 Palaiseau Cedex, France
}

(Received 30 July 2010; revised manuscript received 11 October 2010; published 9 December 2010)

\begin{abstract}
The surface roughness correction to the near-field heat transfer between two rough bulk materials is discussed by using second-order perturbation theory. The results allow for estimating the impact of surface roughness to the heat transfer in recent experiments between two plates and between a microsphere and a plate (using the Derjaguin approximation). Furthermore, we show that the proximity approximation for describing rough surfaces is valid for distances much smaller than the correlation length of the surface roughness even if the heat transfer is dominated by the coupling of surface modes.
\end{abstract}

DOI: 10.1103/PhysRevB.82.245410

PACS number(s): 44.40.+a, 05.40.-a, 41.20.Jb

\section{INTRODUCTION}

Recently, several experimental setups made a measurement of the radiative heat flux on nanoscale feasible. ${ }^{1-4}$ It could be verified by $\mathrm{Hu}$ et al. ${ }^{1}$ that the heat flux between two glass plates exceeds the far-field limit set by Planck's black body radiation law for distances of some microns. Narayanaswamy et $a l .{ }^{2}$ and Shen et $a .^{3}{ }^{3}$ used a different experimental setup measuring the heat flux between microspheres and plates of different materials which allow for detecting the radiative heat flux at much smaller distances, and have reported heat-transfer coefficients three orders of magnitude larger than the black body radiation limit in accordance with theoretical predictions. ${ }^{5,6} \mathrm{~A}$ similar setup was used by Rousseau et l. $^{4}$ for measuring the heat flux between a glass microsphere and a glass sample in a distance regime ranging from $30 \mathrm{~nm}$ to $2.5 \mu \mathrm{m}$. With that experiment the theoretical predictions based on fluctuational electrodynamics ${ }^{7}$ could be verified with high accuracy.

In all these experiments the results have been compared to calculations which do not take into account the roughness of the materials used. Here, we want to tackle the question how the surface roughness affects the heat flux in the near field. Therewith we provide the basis for comparison of the experimental data with theoretical results including roughness effects. Furthermore, our results might be used to study the impact of roughness in thermophotovoltaic devices. ${ }^{8-12}$ The first work considering surface roughness effects for the nearfield heat flux was given by Persson et al. ${ }^{13}$ employing the so-called proximity approximation (PA) (Refs. 14 and 15) to determine the heat flux between two rough surfaces. In a recent work ${ }^{16}$ considering the effect of roughness on the heat flux between a nanoparticle and a rough surface, it has been shown that perturbation theory exactly reproduces the PA for distances smaller than the correlation length of the rough surface.

In this work, we extend the perturbation theory to describe the near-field heat transfer between two semi-infinite media with rough surfaces. This geometry is more suitable for the estimation of surface roughness effects in recent experimental setups. ${ }^{1}$ Utilizing the Derjaguin approximation, our results can also be used to calculate the roughness correction for the experiments of Narayanaswamy et al., ${ }^{2}$ Shen et al. ${ }^{3}$ and Rousseau et $a l .{ }^{4}$ In addition, we show that the PA can be used for vacuum gaps smaller than the correlation length of the surface roughness even when the heat flux is dominant due to the coupling of surface modes. This is an important result since it shows that the PA can indeed be used to obtain a simple estimation of the roughness correction to the heat flux.

The paper is organized as follows: in Sec. II we introduce the mean Poynting vector in terms of transmission coefficients and present in Sec. III the resulting expressions for second-order perturbation theory considering a Gaussian surface roughness. Finally, in Sec. IV we study the impact of surface roughness on the heat flux and give a detailed discussion of the numerical results. In particular, we investigate the range of validity of the PA.

\section{RADIATIVE HEAT TRANSFER}

Let us consider a configuration as depicted in Fig. 1. We have two semi-infinite bodies in local thermal equilibrium at temperatures $T_{1}$ and $T_{2}$ separated by a vacuum gap. In general, both media have different material properties which can be expressed by different permittivities $\epsilon_{1}$ and $\epsilon_{2}$. When considering isotropic and local materials only, the permittivities are scalars and do not depend on the wave vector. Here, we will consider such materials, although, in general, nonlocal effects have to be taken into account. ${ }^{17,18}$

Furthermore, we assume that both bodies have a rough surface expressed by the surface-profile functions $S_{1}(\mathbf{x})$ and $S_{2}(\mathbf{x}+d)$, where $d$ is the mean distance between the two ma-

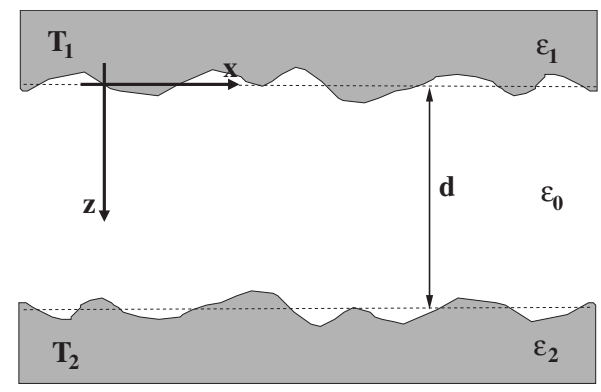

FIG. 1. Sketch of the situation considered here. 
terials and $\mathbf{x}=(x, y)$. Since in this work we focus on stochastic Gaussian surface profiles, ${ }^{19}$ the profile functions fulfill the properties

$$
\begin{gathered}
\left\langle S_{i}(\mathbf{x})\right\rangle=0, \\
\left\langle S_{i}(\mathbf{x}) S_{i}\left(\mathbf{x}^{\prime}\right)\right\rangle=\delta_{i}^{2} W_{i}\left(\left|\mathbf{x}-\mathbf{x}^{\prime}\right|\right) .
\end{gathered}
$$

The brackets \langle\rangle stand for the average over an ensemble of realizations of the surface profiles $S_{i}(\mathbf{x})$ for $i=1,2 ; \delta_{i}$ is the rms height of the surface profiles. The correlation functions $W_{i}\left(\left|\mathbf{x}-\mathbf{x}^{\prime}\right|\right)$ are given by a Gaussian

$$
W_{i}\left(\left|\mathbf{x}-\mathbf{x}^{\prime}\right|\right)=e^{-\left|\mathbf{x}-\mathbf{x}^{\prime}\right|^{2} / a_{i}^{2}}
$$

introducing the transverse correlation length $a_{i}$. In addition we assume that both surface profiles are statistically independent, i.e., $\left\langle S_{1} S_{2}\right\rangle=0$. For the Fourier component $\widetilde{S}_{i}(\boldsymbol{\kappa})$ of the surface-profile functions one obtains for $i=1,2$

$$
\begin{gathered}
\left\langle\tilde{S}_{i}(\boldsymbol{\kappa})\right\rangle=0, \\
\left\langle\widetilde{S}_{i}(\boldsymbol{\kappa}) \tilde{S}_{i}\left(\boldsymbol{\kappa}^{\prime}\right)\right\rangle=(2 \pi)^{2} \delta_{i}^{2} \delta\left(\boldsymbol{\kappa}+\boldsymbol{\kappa}^{\prime}\right) g_{i}(\boldsymbol{\kappa})
\end{gathered}
$$

with the surface roughness power spectra

$$
g_{i}(\kappa)=\int d^{2} x W_{i}(|\mathbf{x}|) e^{-i \boldsymbol{\kappa} \cdot \mathbf{x}}=\pi a_{i}^{2} e^{-\kappa^{2} a_{i}^{2} / 4} .
$$

In order to evaluate the radiative heat transfer between the two bodies, ${ }^{6}$ it suffices to determine the electric Green's dyadic $G\left(\mathbf{r}, \mathbf{r}^{\prime}\right)$ with $\mathbf{r}$ and $\mathbf{r}^{\prime}$ within the vacuum gap. Then the mean Poynting vector is given by ${ }^{6}$

$$
\langle S\rangle=\int \frac{d \omega}{2 \pi}\left[\Theta\left(\omega, T_{1}\right)-\Theta\left(\omega, T_{2}\right)\right]\left\langle S_{\omega}\right\rangle
$$

with

$$
\begin{aligned}
\left\langle S_{\omega}\right\rangle= & 2 \operatorname{Re} \operatorname{Tr}\left[\int d ^ { 2 } x ^ { \prime } \left\langleG\left(\mathbf{r}, \mathbf{r}^{\prime}\right) \partial_{z} \partial_{z^{\prime}} G^{\dagger}\left(\mathbf{r}, \mathbf{r}^{\prime}\right)\right.\right. \\
& \left.\left.-\partial_{z} G^{\dagger}\left(\mathbf{r}, \mathbf{r}^{\prime}\right) \partial_{z^{\prime}} G\left(\mathbf{r}, \mathbf{r}^{\prime}\right)\right\rangle\right]_{\mathbf{r}=\mathbf{r}^{\prime}},
\end{aligned}
$$

where the integration is carried out over a flat surface within the vacuum gap parallel to the $x-y$ plane and

$$
\Theta\left(\omega, T_{i}\right)=\frac{\hbar \omega}{e^{\hbar \omega \beta_{i}}-1}
$$

with $\beta_{i}=1 /\left(k_{\mathrm{B}} T_{i}\right)$ for $i=1,2 ; k_{\mathrm{B}}$ is Boltzmann's constant. Finally, we reformulate Eq. (8) in terms of transmission coefficients. To this end, we first introduce the Fourier representation of the Green's dyadic as

$$
\mathrm{G}\left(\mathbf{r}, \mathbf{r}^{\prime}\right)=\int \frac{d^{2} \boldsymbol{\kappa}}{(2 \pi)^{2}} \int \frac{d^{2} \boldsymbol{\kappa}^{\prime}}{(2 \pi)^{2}} e^{i\left(\boldsymbol{\kappa} \cdot \mathbf{x}-\boldsymbol{\kappa}^{\prime} \cdot \mathbf{x}^{\prime}\right)} \mathrm{G}\left(\boldsymbol{\kappa}, \boldsymbol{\kappa}^{\prime} ; z, z^{\prime}\right)
$$

with $\mathbf{x}=(x, y)$ and the lateral wave vector $\boldsymbol{\kappa}=\left(k_{x}, k_{y}\right)$. Then we find for $\left\langle S_{\omega}\right\rangle$ in Eq. (8)

$$
\left\langle S_{\omega}\right\rangle=\sum_{j, j^{\prime}=s, p} \int \frac{d^{2} \boldsymbol{\kappa}}{(2 \pi)^{2}} \int \frac{d^{2} \boldsymbol{\kappa}^{\prime}}{(2 \pi)^{2}} e^{i\left(\boldsymbol{\kappa}-\boldsymbol{\kappa}^{\prime}\right) \cdot \mathbf{x}}\left\langle T_{j, j^{\prime}}\left(\boldsymbol{\kappa}, \boldsymbol{\kappa}^{\prime} ; \omega\right)\right\rangle,
$$

where

$$
\begin{aligned}
T_{j, j^{\prime}}\left(\boldsymbol{\kappa}, \boldsymbol{\kappa}^{\prime} ; \omega\right)= & 2 \operatorname{Re} \operatorname{Tr} \int \frac{d^{2} \boldsymbol{\kappa}^{\prime \prime}}{(2 \pi)^{2}}\left[\mathrm{G}\left(\boldsymbol{\kappa}, \boldsymbol{\kappa}^{\prime \prime}\right) \partial_{z} \partial_{z^{\prime}} \mathrm{G}^{\dagger}\left(\boldsymbol{\kappa}^{\prime}, \boldsymbol{\kappa}^{\prime \prime}\right)\right. \\
& \left.-\partial_{z} \mathrm{G}^{\dagger}\left(\boldsymbol{\kappa}, \boldsymbol{\kappa}^{\prime \prime}\right) \partial_{z^{\prime}} \mathrm{G}\left(\boldsymbol{\kappa}^{\prime}, \boldsymbol{\kappa}^{\prime \prime}\right)\right]_{z=z^{\prime}}
\end{aligned}
$$

is the transmission coefficient. It describes the transmission of a thermally emitted wave with wave vector $\boldsymbol{\kappa}$ and polarization $j$ from body 1 to body 2 which is scattered into wave vector $\boldsymbol{\kappa}^{\prime}$ and polarization $j^{\prime}$ during the transmission process. By means of the transmission coefficient the Poynting vector in Eq. (7) can be converted into a Landauer-type form as was shown in Ref. 20. After ensemble averaging, the translational and rotational symmetries are restored so that the mean transmission coefficient reduces to

$$
\left\langle T_{j, j^{\prime}}\left(\boldsymbol{\kappa}, \boldsymbol{\kappa}^{\prime} ; \omega\right)\right\rangle=(2 \pi)^{2} \delta\left(\boldsymbol{\kappa}-\boldsymbol{\kappa}^{\prime}\right) \delta_{j, j^{\prime}} T_{j}(\boldsymbol{\kappa} ; \omega)
$$

and hence

$$
\left\langle S_{\omega}\right\rangle=\sum_{j=s, p} \int \frac{d^{2} \kappa}{(2 \pi)^{2}} T_{j}(\boldsymbol{\kappa} ; \omega) .
$$

In the following section we will determine the perturbation expansion of the transmission coefficient $T_{j}=T_{j}^{(0)}+T_{j}^{(1)}+T_{j}^{(2)}$ $+\cdots$ and $\left\langle S_{\omega}\right\rangle=S_{\omega}^{(0)}+\left\langle S_{\omega}^{(1)}\right\rangle+\left\langle S_{\omega}^{(2)}\right\rangle+\cdots$.

\section{PERTURBATION RESULT}

By using Eqs. (12)-(14) we obtain the perturbation expansion for the mean Poynting vector by expanding the Green's dyadic with respect to the surface-profile functions $S_{1}$ and $S_{2}$. A short discussion of the perturbation method ${ }^{21}$ used here can be found in Ref. 22. Note that, this perturbation approach has also been used to study for example the roughness effects for the Casimir force. ${ }^{23,24}$ We remark that the perturbation theory can be applied as far as the rms of the surface profiles is the smallest length scale of the problem. ${ }^{25}$

Then, by inserting the zeroth-order dyadic Green's function from Ref. 22 into the expression for the mean transmission coefficient in Eq. (12) one retrieves for $\left\langle S_{\omega}\right\rangle$ from Eq. (14) the well-known result, ${ }^{7}$ for the case of two media with flat surfaces

$$
T_{j}^{(0)}(\kappa ; \omega)= \begin{cases}\frac{\left(1-\left|r_{j}^{1}\right|^{2}\right)\left(1-\left|r_{j}^{2}\right|^{2}\right)}{\left|D_{j j}\right|^{2}} & \kappa \leq k_{0} \\ \frac{4 \operatorname{Im}\left(r_{j}^{1}\right) \operatorname{Im}\left(r_{j}^{2}\right) e^{-2 \gamma d}}{\left|D_{j j}\right|^{2}} & \kappa>k_{0},\end{cases}
$$

where $\gamma^{2}=\kappa^{2}-k_{0}^{2}, k_{0}=\omega / c$, and $r_{j}^{i}$ (for $i=1,2$ and $j=s, p$ ) are Fresnel's reflection coefficients of the two bodies for $s$ or $p$ polarization; $D_{j j}=1-r_{j}^{1} r_{j}^{2} \exp \left(2 i \gamma_{\mathrm{r}} d\right)$ is the Fabry-Pérottype denominator, where $\gamma_{\mathrm{r}}^{2}=k_{0}^{2}-\kappa^{2} . T_{j}^{(0)}$ is the transmission coefficient between the two bodies with flat surfaces having values between zero and one. In the propagating regime 
$\left(\kappa<k_{0}\right)$ the property $T_{j}^{(0)} \leq 1$ means that the heat flux is always smaller than that between two black bodies for which $T_{j}^{(0)}=1$. In the evanescent regime the maximal value of one can be achieved for such pairs $(\omega, \kappa)$ which fulfill the dispersion relation of the coupled surface modes of the two surfaces. $^{26}$

To get the first nonvanishing term for the surface roughness correction, it is necessary to expand the Green's functions up to second order since $\left\langle G^{(1)}\right\rangle=0$ and therefore, $T_{j}^{(1)}$ $=\left\langle S_{\omega}^{(1)}\right\rangle=0$. The resulting second-order correction can be written as

$$
\left\langle S_{\omega}^{(2)}\right\rangle=S_{\omega, \mathrm{diff}}^{(2)}+S_{\omega, \mathrm{spec}}^{(2)}=\sum_{j=\mathrm{s}, \mathrm{p}} \int \frac{d^{2} \kappa}{(2 \pi)^{2}}\left(T_{j, \mathrm{diff}}^{(2)}+T_{j, \mathrm{spec}}^{(2)}\right),
$$

where we have splitted the result into the specular part which depends on the mean field or mean Green's function only and the so-called diffuse part which is per definition given by $\left\langle S_{\omega}^{(2)}\right\rangle-S_{\omega, \text { spec }}^{(2)}$. For the diffuse part, we obtain ${ }^{22}$

$$
\begin{aligned}
\sum_{j=\mathrm{s}, \mathrm{p}} T_{j, \mathrm{diff}}^{(2)}= & -\int \frac{d^{2} \boldsymbol{\kappa}^{\prime}}{(2 \pi)^{2}} k_{0}^{2}\left|\epsilon_{1}-1\right|^{2} \frac{g_{1}\left(\left|\boldsymbol{\kappa}-\boldsymbol{\kappa}^{\prime}\right|\right)\left(k_{0} \delta_{1}\right)^{2}}{4\left|\gamma_{\mathrm{r}}\right|^{2}\left|\gamma_{\mathrm{r}}^{\prime}\right|^{2}} \\
& \times\left[Q_{\mathrm{s}}^{2} Q_{\mathrm{s}}^{2 \prime}\left(\hat{\boldsymbol{\kappa}} \cdot \hat{\boldsymbol{\kappa}}^{\prime}\right)^{2}+Q_{\mathrm{s}}^{2} Q_{\mathrm{p}}^{2 \prime} \frac{\left|\gamma_{1}^{\prime}\right|^{2}}{\left|\epsilon_{1}\right| k_{0}^{2}}\left(\hat{\boldsymbol{\kappa}} \times \hat{\boldsymbol{\kappa}}^{\prime}\right)^{2}\right. \\
& +Q_{\mathrm{p}}^{2} Q_{\mathrm{s}}^{2 \prime}\left(\hat{\boldsymbol{\kappa}} \times \hat{\boldsymbol{\kappa}}^{\prime}\right)^{2} \frac{\left|\gamma_{1}\right|^{2}}{\left|\epsilon_{1}\right| k_{0}^{2}} \\
& \left.+Q_{\mathrm{p}}^{2} Q_{\mathrm{p}}^{2 \prime} \frac{\left|\boldsymbol{\kappa} \boldsymbol{\kappa}^{\prime} \epsilon_{1}-\hat{\boldsymbol{\kappa}} \cdot \hat{\boldsymbol{\kappa}}^{\prime} \gamma_{1} \gamma_{1}^{\prime}\right|^{2}}{\left|\epsilon_{1}\right|^{2} k_{0}^{4}}\right]+(1 \leftrightarrow 2)
\end{aligned}
$$

where $t_{s / p}^{i}$ for $i=1,2$ are the amplitude transmission coefficients of the two interfaces for $s$ - and $p$-polarized modes; $\gamma_{i}^{2}=k_{0}^{2} \epsilon_{i}-\kappa^{2}(i=1,2), \hat{\boldsymbol{\kappa}}=\left(k_{x}, k_{y}\right) / \kappa$ and $(1 \leftrightarrow 2)$ symbolizes the term obtained by interchanging the index 1 and 2 . The functions $Q_{s / p}^{1 / 2}$ are defined as

$$
Q_{s / p}^{1 / 2}=\frac{\left|t_{s / p}^{2 / 1}\right|^{2}}{\left|D_{s s / p p}\right|^{2}}\left[\operatorname{Re}\left(\gamma_{\mathrm{r}}\right)\left(1-\left|r_{p / s}^{1 / 2}\right|^{2}\right)+2 \operatorname{Im}\left(\gamma_{\mathrm{r}}\right) \operatorname{Im}\left(r_{s / p}^{1 / 2}\right) e^{-2 \gamma d}\right]
$$

It is seen from Eq. (17) that the diffuse correction to the transmission coefficient and therefore, also to $S_{\omega, \text { diff }}^{(2)}$ is always negative. Hence, due to the diffuse scattering in the rough surface the transmission coefficient becomes smaller and therefore, the heat transfer less efficient.

For the specular contribution to the mean transmission coefficient we obtain ${ }^{22}$ for propagating modes $\left(\kappa \leq k_{0}\right)$

$$
\begin{aligned}
T_{j, \mathrm{spec}}^{(2)}= & -\frac{\left(1-\left|r_{j}^{2}\right|^{2}\right)}{\left|D_{j j}\right|^{2}} \operatorname{Im}\left[\left(r_{j}^{1}-r_{j}^{2 *} e^{-2 i \gamma_{\mathrm{r}} d}\right) \frac{\left(t_{j}^{1 *}\right)^{2} h_{j}^{1 *} A_{1, j i}^{*}}{\gamma_{\mathrm{r}}}\right] \\
& +(1 \leftrightarrow 2)
\end{aligned}
$$

$$
\begin{aligned}
T_{j, \mathrm{spec}}^{(2)}= & \frac{2 \operatorname{Im}\left(r_{j}^{2}\right)}{\left|D_{j j}\right|^{2}} \operatorname{Im}\left[\left(1-r_{j}^{1} r_{j}^{2 *} e^{-2 \gamma d}\right) \frac{\left(t_{j}^{1}\right)^{2} h_{j}^{1} A_{1, j j}}{\gamma}\right] e^{-2 \gamma d} \\
& +(1 \leftrightarrow 2) .
\end{aligned}
$$

The expression for $A_{1 / 2, s s}$ and $A_{1 / 2, p p}$ can be found in Ref. 22 and $h_{s}^{1 / 2}=1, h_{p}^{1 / 2}=\epsilon_{1 / 2}$. By comparing the specular secondorder transmission coefficients with the zeroth-order expressions, it becomes apparent that they are very similar to $T_{j}^{(0)}$, but with the difference that one of the terms $1-\left|r_{j}^{1 / 2}\right|$ or $2 \operatorname{Im}\left(r_{j}^{1 / 2}\right)$ is replaced by a much more complex term involving $A_{1 / 2, j j}$. By means of this term the roughness scattering within one of the two surfaces 1 or 2 is taken into account yielding a roughness correction to the transmission which can be either positive or negative.

In general, within second-order perturbation theory the diffuse and the specular components describe the correction to the heat flux due to the scattering within only one of the rough surfaces since both expressions split up into the sum of a term proportional to $\delta_{1}^{2}$ and $\delta_{2}^{2}$. The different scattering processes can be classified as follows: The zeroth-order scattering is the specular scattering of the two flat mean surfaces. The diffuse component describes the correction to the heat flux for first-order scattering of an incoming wave with lateral wave vector $\boldsymbol{\kappa}$ into a wave with $\boldsymbol{\kappa}^{\prime}$. For such scattering processes, incoming $s$-polarized waves can be scattered into $p$-polarized waves and propagating waves into evanescent waves and vice versa. On the other hand, the specular component describes the correction to the heat flux due to a second-order scattering of an incoming wave with $\boldsymbol{\kappa}$ into a wave with $\boldsymbol{\kappa}^{\prime \prime}=\boldsymbol{\kappa}$, since it describes the scattering of the mean field. These scattering processes can be divided into two different types: a direct second-order scattering originating from terms proportional to $\widetilde{S}_{i}^{(2)}\left(\boldsymbol{\kappa}-\boldsymbol{\kappa}^{\prime \prime}\right)$ and an indirect second-order scattering through intermediate states with wave vectors $\boldsymbol{\kappa}^{\prime}$ originating from terms proportional to $\widetilde{S}_{i}^{(1)}\left(\boldsymbol{\kappa}-\boldsymbol{\kappa}^{\prime}\right) \widetilde{S}_{i}^{(1)}\left(\boldsymbol{\kappa}^{\prime}-\boldsymbol{\kappa}^{\prime \prime}\right)$. The latter one is a sequence of two first-order scattering processes as for the diffuse scattering but with the constraint that the polarization and the lateral wave vector are the same before and after the scattering sequence.

\section{NUMERICAL RESULTS AND DISCUSSION}

In the following we discuss the impact of surface roughness on the near-field heat transfer numerically. We use the material properties of $\mathrm{SiC}$ and $\mathrm{Au}$ for both media with parameters taken from Refs. 17 and 27 and set $T_{2}=0 \mathrm{~K}$ and $T_{1}=300 \mathrm{~K}$. Additionally, we choose the surface roughness parameters $\delta_{i}=5 \mathrm{~nm}$ and $a_{i}=200 \mathrm{~nm}$ for $i=1,2$, i.e., we consider a very shallow surface roughness with $\delta_{i} / a_{i}$ $=0.025$.

\section{A. Distance dependence}

First, we turn to the distance dependence of the roughness correction to the near-field heat transfer. To this aim, we start with a plot of the heat flux between two bodies with flat surfaces in Fig. 2(a) considering two SiC and two Au slabs.

and for evanescent modes $\left(\kappa>k_{0}\right)$ 

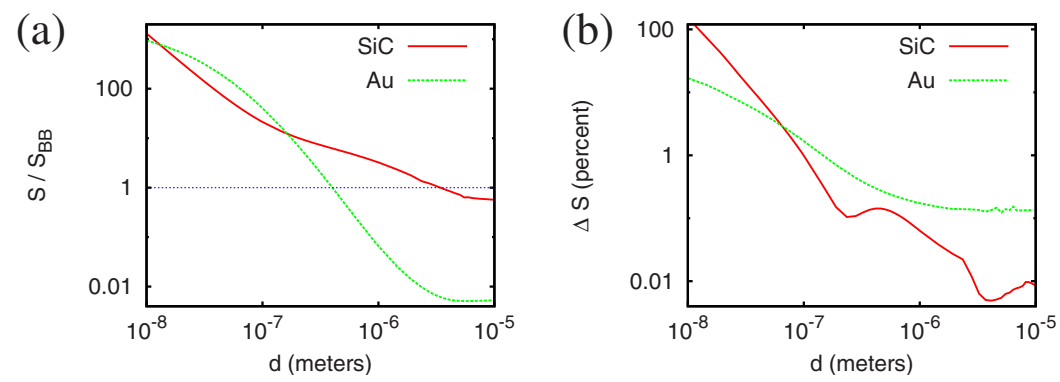

FIG. 2. (Color online) Plot of (a) $S^{(0)}$ over distance for two $\mathrm{SiC}$ plates and two $\mathrm{Au}$ plates normalized to the black body value $S_{\mathrm{BB}}$ with (b) the corresponding roughness correction $\Delta S$.
$\mathrm{SiC}$ has a surface phonon resonance, whereas $\mathrm{Au}$ has no surface-plasmon resonance in the infrared region. One can see that the curves for the $\mathrm{SiC}$ and the $\mathrm{Au}$ plates are quite different. For $\mathrm{SiC}$ the heat transfer is relatively large in the propagating regime $\left(d>\lambda_{\mathrm{th}}=\hbar \beta c=7.68 \mu \mathrm{m}\right.$ for $\left.T=300 \mathrm{~K}\right)$ due to the low reflectivity of this material and has values close to the black body limit $S_{\mathrm{BB}}=459.3 \mathrm{Wm}^{-2}$, whereas for $\mathrm{Au}$ which has a high reflectivity the heat flux is very small. In the evanescent regime $\left(d<\lambda_{\text {th }}\right)$ the heat flux is for $\mathrm{SiC}$ dominated by the $p$-polarized surface mode contribution giving values which can exceed the black body result by more than three orders of magnitude for the here chosen distance regime. On the other hand, for the Au plates the heat flux is dominated by the $s$-polarized contribution and the large amount of transferred heat can be attributed to induced Foucault currents. ${ }^{17}$ For distances smaller than $100 \mathrm{~nm}$ the curve for $\mathrm{SiC}$ becomes proportional to $1 / d^{2}$ which is the wellknown result in the quasistatic limit. ${ }^{28,29}$

Now, we have a look on the impact of surface roughness. In Fig. 2(b) we plot the roughness correction to the heat transfer $\Delta S=\left\langle S^{(2)}\right\rangle / S^{(0)}$. First of all, for both materials the roughness correction is positive for all distances so that roughness increases the heat flux with respect to the case of flat surfaces. In the propagating regime the relative correction is very small (smaller than 0.01 percent) for $\mathrm{SiC}$ and is limited due to the fact that even if we introduce roughness the overall amount of thermal radiation cannot be larger than $S_{\mathrm{BB}}$. For Au the relative correction is one order-of-magnitude larger, but the absolute correction is still very small, since $S^{(0)}$ has values smaller than $0.01 S_{\mathrm{BB}}$. In the opposite limit of small distances, i.e., in the evanescent regime the relative correction due to roughness can become large. For example, for $\mathrm{SiC}$ the relative correction is about $8 \%$ for $d=40 \mathrm{~nm}$. Since for that distance the heat flux is as large as 91 times the black body value, this is an increase of about seven times $S_{\mathrm{BB}}$. For the Au plates we find a roughness correction of $5 \%$ for $d=40 \mathrm{~nm}$. At this distance $S^{(0)}$ is about 223 times the black body value yielding an absolute correction of about 11 times $S_{\mathrm{BB}}$.

Finally, in Fig. 3 we plot the specular and diffuse part of the roughness correction for $\mathrm{SiC}$ only. Keeping in mind that the diffuse part is purely negative in this case, whereas we find that the specular part is purely positive. It can be seen that for distances larger than $500 \mathrm{~nm}$ the diffuse part is negligibly small compared to the specular one, whereas for distances smaller than $500 \mathrm{~nm}$ both contributions have to be accounted for. Obviously, the specular part tends to overestimate the roughness correction for such distances and only when also considering the diffuse contribution one will get the correct result.
To understand why the diffuse part becomes important for small distances we take a closer look at the purely evanescent components of the diffuse Poynting vector in Eqs. (16) and (17). For the scattering of evanescent $p$ modes into evanescent $p$ modes this component can be written as

$$
\begin{aligned}
S_{\omega, \text { diff }}^{(2)}= & -\int_{\kappa>k_{0}} d^{2} \boldsymbol{\kappa} \int_{\kappa^{\prime}>k_{0}} d^{2} \boldsymbol{\kappa}^{\prime} Q_{p}^{1} g_{2}\left(\left|\boldsymbol{\kappa}-\boldsymbol{\kappa}^{\prime}\right|\right) \\
& \times f_{p p}\left(\boldsymbol{\kappa}, \boldsymbol{\kappa}^{\prime} ; \omega\right) Q_{p}^{1 \prime}+(1 \leftrightarrow 2),
\end{aligned}
$$

where we have introduced the function $f_{p p}$ comprising all the factors which are not essential for the discussion of the integral and

$$
Q_{p}^{1}=\frac{\left|t_{p}^{2}\right|^{2}}{\left|D_{p p}\right|^{2}} 2 \gamma \operatorname{Im}\left(r_{p}^{1}\right) e^{-2 \gamma d} .
$$

The quantity $Q_{p}^{1}$ is proportional to the transmission coefficient $T_{p}^{(0)}$ for evanescent $p$ modes coming from slab 1 which are transmitted into slab 2 and conversely. This means that the integrand is proportional to $T_{p}^{(0)}(\kappa) g_{2}\left(\left|\boldsymbol{\kappa}-\boldsymbol{\kappa}^{\prime}\right|\right) T_{p}^{(0)}\left(\boldsymbol{\kappa}^{\prime}\right)$ describing the transmission of a $p$-polarized evanescent wave with wave vector $\boldsymbol{\kappa}$ from slab 1 to slab 2 being scattered into a $p$-polarized evanescent wave with wave vector $\boldsymbol{\kappa}^{\prime}$ which is transmitted back into slab 1. Therefore, this product is proportional to the transmission coefficient describing how much of the energy is scattered back into the first slab by diffuse scattering within the second slab (see Fig. 4). In the nonretarded or quasistatic limit the weighting factor $\gamma \exp (-2 \gamma d)$ for $Q_{p}^{1}$ becomes $\kappa \exp (-2 \kappa d)$, i.e., the transmission is the strongest if $\kappa \approx 1 / d$. Hence, one can conclude that the integrand is large when $\kappa \approx \kappa^{\prime} \approx 1 / d$. It follows that for $\left|\boldsymbol{\kappa}-\boldsymbol{\kappa}^{\prime}\right| \gg 1 / d$ the integrand is small. Since the scattering within the rough surface is approximately limited to

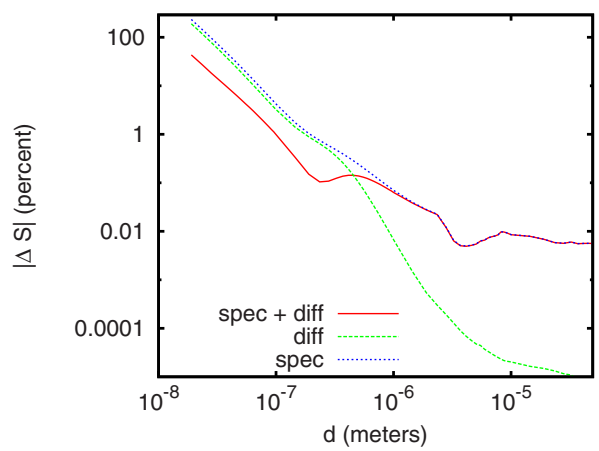

FIG. 3. (Color online) Plot of the specular and diffuse part of the roughness correction $|\Delta\langle S\rangle|$ over distance for SiC. 


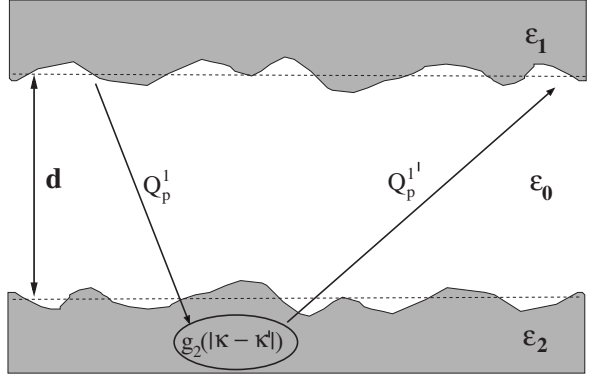

FIG. 4. Sketch of the diffuse scattering in terms of transmission coefficients. Here $Q_{p}^{1} \propto T_{p}^{(0)}(\kappa)$ and $Q_{p}^{1 \prime} \propto T_{p}^{(0)}\left(\kappa^{\prime}\right)$.

$a_{i}\left|\boldsymbol{\kappa}-\boldsymbol{\kappa}^{\prime}\right| \leq 1$ by the surface roughness power spectrum [see Eq. (6)], we can conclude that for $a_{i} \ll d$ the diffuse component $S_{\omega, \text { diff }}^{(2)}$ is comparatively small and can get large in the opposite limit, i.e., for $d \ll a_{i}$. In addition, due to the FabryPérot denominators in $Q_{\mathrm{p}}^{1}$ and $Q_{\mathrm{p}}^{1 \prime}$ the diffuse contribution is especially large if $\kappa$ and $\kappa^{\prime}$ corresponds to surface modes. Such scattering processes with $\kappa \approx \kappa^{\prime}$ are reminiscent of directional scattering of surface modes. ${ }^{30,31}$

\section{B. Transmission coefficient in the $(\omega, \kappa)$ plane}

To get some insight into the physical mechanism underlying the roughness correction, we will first discuss the transmission coefficients $T_{j}^{(0)}$ and $T_{j}^{(2)}$ which are defined in Eqs. (15), (17), (19), and (20) and then we will turn to the spectrum of the zeroth-order Poynting vector $S_{\omega}^{(0)}$ and the secondorder term $\left\langle S_{\omega}^{(2)}\right\rangle$. First, for distances larger or comparable to the thermal wavelength $\lambda_{\text {th }} \approx 7.68 \mu \mathrm{m}$ at $300 \mathrm{~K}$ the propagating modes with $\kappa \leq k_{0}$ dominate the contribution to the mean Poynting vector. For the two slab configuration these modes are given by the gap modes for which $T_{j}^{(0)}$ equals 1 as shown in Fig. 5(a) for $p$-polarized modes choosing $d=5 \mu \mathrm{m}$. The correction to the transmission coefficient due to roughness scattering is in this case dominated by the specular part. Nonetheless, this roughness correction is rather
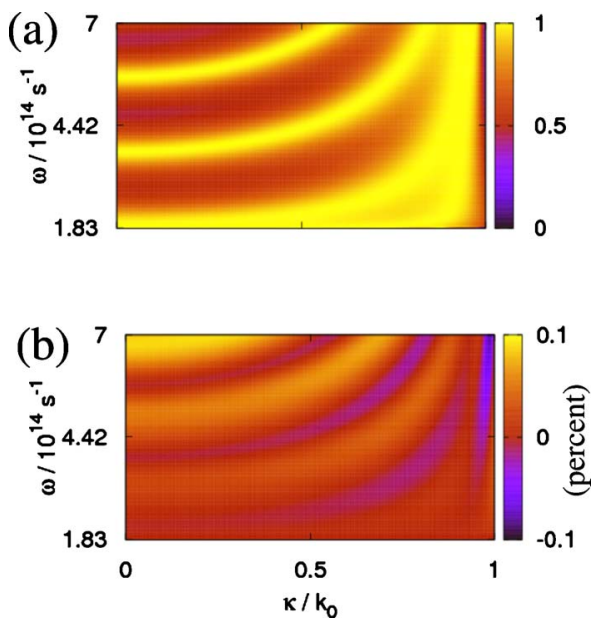

FIG. 5. (Color online) Plot of (a) $T_{p}^{(0)}$ as defined in Eq. (15) and (b) $T_{p \text {,spec }}^{(2)} / T_{p}^{(0)}$ in the propagating regime using $d=5 \mu \mathrm{m}$ for frequencies ranging from $\omega=\omega_{1}=1.83 \times 10^{14}$ to $7 \times 10^{14} \mathrm{~s}^{-1}$. (a)

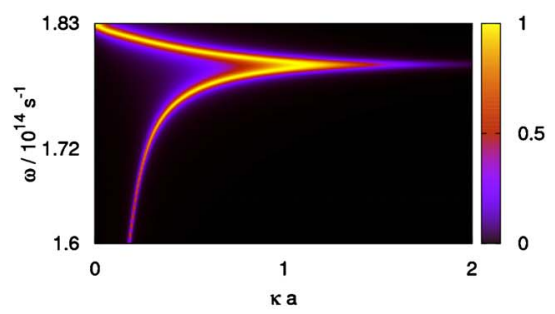

(b)

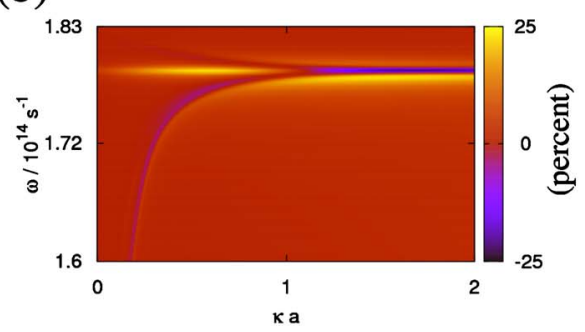

(c)

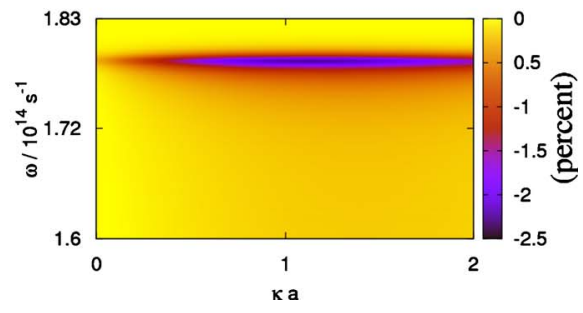

FIG. 6. (Color online) Plot of (a) $T_{p}^{(0)}$, (b) $T_{p \text {,spec }}^{(2)} / T_{p}^{(0)}$, and (c) $T_{p \text {,diff }}^{(2)} / T_{p}^{(0)}$ using $d=500 \mathrm{~nm}$.

small for the chosen roughness parameters as illustrated in Fig. 5(b).

Apart from this, due to the roughness scattering the evanescent surface polariton contribution can couple to the propagating modes ${ }^{16}$ so that even for distances $d>\lambda_{\text {th }}$ one finds a relatively large correction to the transmission coefficient for frequencies close to the surface resonance frequency, which is for $\mathrm{SiC}$ given by $\omega_{\mathrm{SPhP}}=1.787 \times 10^{14} \mathrm{~s}^{-1}$. For $d=5 \mu \mathrm{m}$ this correction is in the order of some percent. Since the propagating modes dominate the heat transfer for $d=5 \mu \mathrm{m}$ the overall correction to the Poynting vector is small.

Now, for distances much smaller than the thermal wavelength $\lambda_{\text {th }}$, the near-field heat transfer is solely determined by the $p$-polarized surface mode contribution. For such distances the diffuse contribution cannot be neglected. Choosing a distance of $d=500 \mathrm{~nm}$ we show in Fig. 6(a) a plot of $T_{p}^{(0)}$ first. Here, one can observe the splitting of the two surface phonon polariton branches. ${ }^{31,32}$ Furthermore, one can estimate from Fig. 6(a) that the relevant contributions stem from lateral wave-vectors $\kappa$ smaller than about $1.5 / a$. In Fig. 6(b) we show the corresponding plot of the roughness correction $T_{p \text {,spec }}^{(2)} / T_{p}^{(0)}$. We find a qualitative similar correction as found for the roughness scattering considering only one rough surface ${ }^{16}$ with the difference (apart from the splitting of the surface mode branches) that there is a positive correction for lateral wave vectors smaller than about $1 / a$ for fre- 


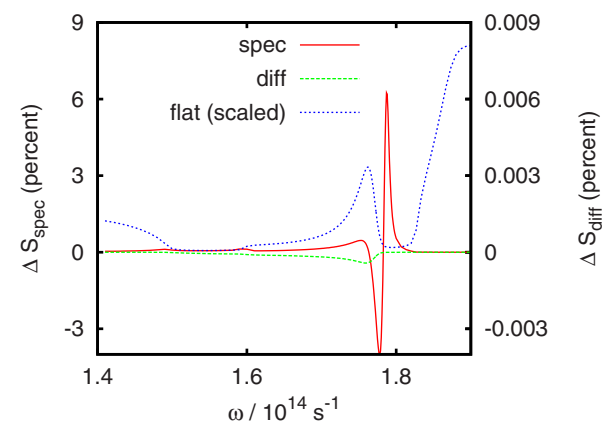

FIG. 7. (Color online) Plot of $\left\langle S_{\omega}^{(2)}\right\rangle / S_{\omega}^{(0)}$ in a small frequency range around the surface phonon frequency of $\mathrm{SiC}\left(\omega_{\mathrm{SPhP}}=1.787\right.$ $\times 10^{14} \mathrm{~s}^{-1}$ ) using $d=5 \mu \mathrm{m}$. We have plotted the specular (solid line/red line) and the diffuse (dashed line/green line) contributions (note the different scales at the right and left-hand side) and $S_{\omega}^{(0)}$ (dotted line/blue line).

quencies around $\omega_{\mathrm{SPhP}}$ and again a negative correction for lateral wave-vectors larger than $1 / a$. As we will see below, when integrating $T_{p}^{(2)}$ over the lateral wave vector to get $\left\langle S_{\omega}^{(2)}\right\rangle$ this positive correction will make the negative one weaker compared to the case of only one rough surface in Ref. 16. This results in a positive overall correction to the heat flux for all distances in contrast to the results found for only one rough surface. ${ }^{16}$

In Fig. 6(c) we plot the corresponding diffuse contribution $T_{p \text {,diff }}^{(2)} / T_{p}^{(0)}$ in the same $(\omega, \kappa)$ range. As expected the diffuse component gives a negative correction which is relatively large for frequencies near the surface mode resonance. This correction can be as large as $2.5 \%$ for $d=500 \mathrm{~nm}$. Hence, we can conclude that in the evanescent regime, where the heat transfer is due to the coupling of surface phonon polaritons the roughness correction can be relatively large even for a shallow surface roughnesses.

\section{Spectral roughness correction}

Now, we turn to the effect of roughness scattering to the Poynting vector in the spectral domain, i.e., we concentrate on $\left\langle S_{\omega}^{(2)}\right\rangle / S_{\omega}^{(0)}$. In Fig. 7 we have plotted $\left\langle S_{\omega}^{(2)}\right\rangle / S_{\omega}^{(0)}$ for the specular and diffuse contribution using $d=5 \mu \mathrm{m}$ in a frequency range between $1.4 \times 10^{14}$ and $2 \times 10^{14} \mathrm{~s}^{-1}$. We have also plotted $S_{\omega}^{(0)}$ using a scaling such that the curve fits into the plot. First of all one can see that, for such a large distance, the diffuse contribution is negative as implied by Eq. (17) and about three orders of magnitude smaller than the specular contribution. On the other hand, the specular contribution gives a relatively large correction of some percent near the surface resonance $\omega_{\mathrm{SPhP}}=1.787 \times 10^{14} \mathrm{~s}^{-1}$, only. This correction is the sum of the positive and negative corrections to the propagating gap mode and the evanescent and propagating polariton mode correction within the reststrahlen region. Since the positive contribution to $\left\langle S_{\omega}^{(2)}\right\rangle / S_{\omega}^{(0)}$ is larger than the negative one, one can expect to get a purely positive roughness correction to the Poynting vector when carrying out the frequency integral. Furthermore, since the heat flux is not solely determined by the surface mode, but rather dominated by the gap modes (for frequencies greater than $\omega_{l}$ ) for

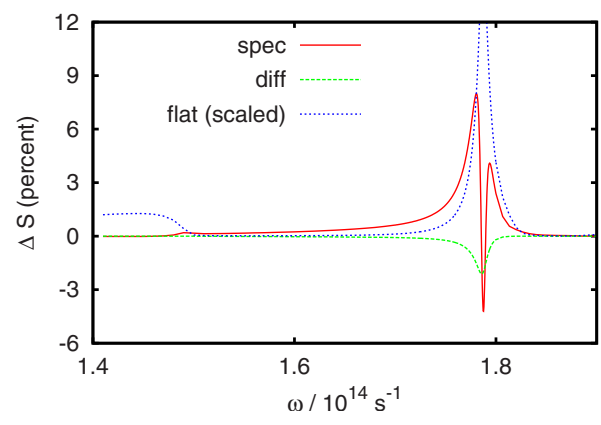

FIG. 8. (Color online) As Fig. 7 using $d=500 \mathrm{~nm}$.

which the roughness correction is very small, it follows that the roughness correction to the Poynting vector is small for distances around or greater than the thermal wavelength.

In the evanescent regime, i.e., for a distance of $d$ $=500 \mathrm{~nm}$ we show in Fig. 8 the correction due to the scattering of surface phonon polaritons. As in the case for one rough surface ${ }^{16}$ we find for the specular part a positive correction for frequencies below and above the surface phonon polariton frequency $\left(\omega_{\mathrm{SPh}}=1.787 \times 10^{14} \mathrm{~s}^{-1}\right)$ and a negative one for frequencies very close to the $\omega_{\mathrm{SPhP}}$. In contrast to the case of one rough surface, here the positive contributions are larger than the negative ones so that the overall Poynting vector is positive at this distance. Furthermore, the diffuse contribution to the heat flux starts to play an important role and gives contributions in the same order of magnitude as the specular one for frequencies near $\omega_{\mathrm{SPhP}}$. This negative correction due to the diffuse component is still so small that the integral over all frequencies remains positive. Therefore, for the configuration considered here, the scattering of surface phonon polaritons does not imply a negative correction to the heat flux as it was found for the heat transfer between a nanoparticle and a rough surface. ${ }^{16}$ In addition, since the zeroth order $S_{\omega}^{(0)}$ is dominated by the surface mode contribution for distances much smaller than $\lambda_{\text {th }}$, the roughness correction of several percent gives a change in the heat flux of several percent. Hence, in the evanescent regime one can expect that the roughness correction will be relatively large compared to the propagating regime.

\section{Proximity approximation $\left(d \ll \min \left\{a_{1}, a_{2}\right\}\right)$}

Now, we want to compare the numerical results for the heat flux with the so-called PA. This will be useful to understand why the coherent flux is increased by the roughness. Within this approximation the rough surfaces are locally approximated by a flat surface (see Fig. 9) so that the mean flux

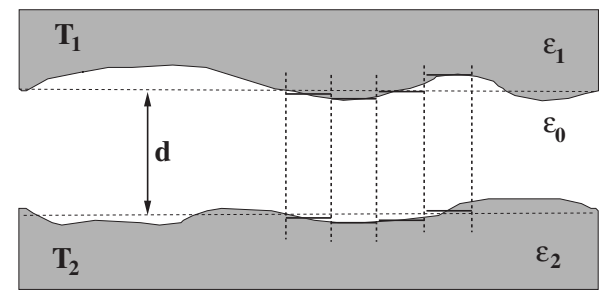

FIG. 9. Sketch of the proximity approximation. 


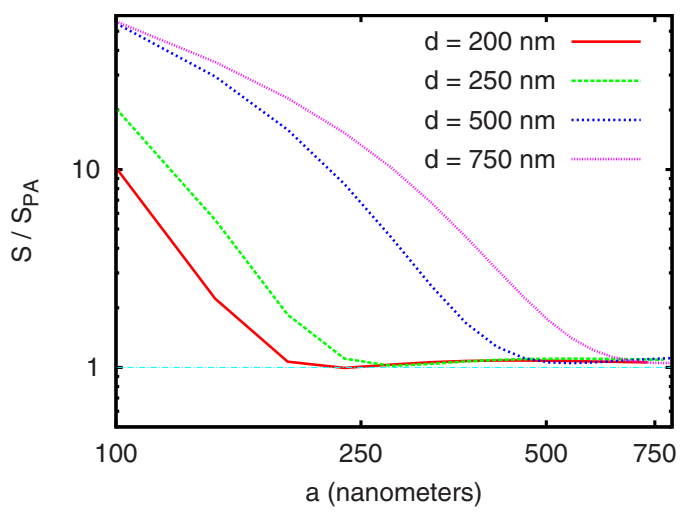

FIG. 10. (Color online) Plot of $S(d) / S_{\mathrm{PA}}(d)$ for SiC over $a$ keeping the rms constant at $\delta=5 \mathrm{~nm}$ for $d=200,250,500$, and $750 \mathrm{~nm}$.

can be computed from the flux between two flat surfaces $S^{(0)}$ by

$$
\begin{aligned}
\langle S(d)\rangle & \approx\left\langle S^{(0)}\left[d-S_{1}(\mathbf{x})+S_{2}(\mathbf{x})\right]\right\rangle \\
& =S^{(0)}(d)+\frac{1}{2} \frac{\partial^{2}}{\partial d^{2}} S^{(0)}(d)\left(\delta_{1}^{2}+\delta_{2}^{2}\right)+\mathcal{O}(4) \\
& =S_{\mathrm{PA}} .
\end{aligned}
$$

When considering dielectric materials, then $S^{(0)} \propto d^{-2}$ in the quasistatic regime so that the PA can be further simplified to

$$
S_{\mathrm{PA}} \approx S^{(0)}\left[1+3 \frac{\left(\delta_{1}^{2}+\delta_{2}^{2}\right)}{d^{2}}\right] .
$$

For the case of two rough surfaces the PA was first used to estimate the near-field radiative heat transfer between two rough surfaces in Ref. 13.

Now, with the perturbation result we can discuss the range of validity of this very simple approximation. As for the case of one rough surface discussed in Ref. 16 one can expect that the PA is valid as far as $d \ll \min \left\{a_{1}, a_{2}\right\}$ since then the local approximation by flat surfaces with a lateral extension much smaller than $a_{i}$ can be assumed to be useful. In order to explore the range of validity of the PA we show in Fig. 10 a plot of $S(d) / S_{\mathrm{PA}}(d)$ over the correlation length $a_{1}=a_{2}=a$ for different distances. It can be seen that the ratio $S(d) / S_{\mathrm{PA}}(d)$ goes approximately to 1 for $a>d$. Hence, we can conclude that the PA can be used for $d<a$.

This conclusion is not trivial since for $\mathrm{SiC}$ the near-field heat transfer is due to the coupling of thermally excited surface modes. The thermal near fields associated with these surface modes can have a lateral coherence length $l_{\text {coh }}$ larger than the vacuum wavelength ${ }^{5,33}$ and can therefore, be larger than the correlation length $a$ considered here. However, in the quasistatic regime $\left(d \ll \lambda_{\text {th }}\right)$ it is found ${ }^{34,35}$ that $l_{\text {coh }} \approx d$. Hence, for distances $d \ll a$ the thermally generated fields above different surface elements having an area smaller than $a \times a$ and larger than $d \times d$ are uncorrelated. Hence, for $d$ $\ll a$ the flat areas can indeed be regarded as independent so that the PA is valid.

Numerically, we find for $\mathrm{SiC}$ that for distances $d \ll a$ the correction to the heat flux is given by the sum of the positive specular and the negative diffuse contribution. The specular

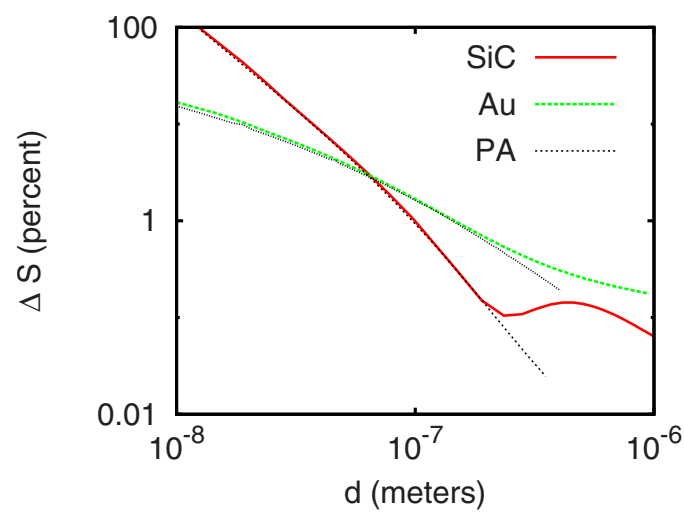

FIG. 11. (Color online) As Fig. 2(b) but with the PA for SiC and Au calculated from $S^{(0)}$ with Eq. (23).

correction tends to give results larger than the one predicted by the PA. Only, when also considering the diffuse component of the heat flux, we retrieve the PA result. Therefore, the interplay of the diffuse and the specular scattering of the surface polaritons guarantees the validity of the PA. On the other hand, for $\mathrm{Au}$ which has no surface resonance around $\lambda_{\text {th }}$ we find that the diffuse part is negligible small. In this case the PA is also valid and is due to the specular part only.

In Fig. 11 we plot the roughness correction for $\mathrm{SiC}$ and $\mathrm{Au}$ together with a numerical evaluation of the PA in Eq. (23). In both cases the PA gives a good approximation for distances $d \ll a$. As can be expected the curve for $\mathrm{SiC}$ converges for very small distances to the quasistatic expression for the PA in Eq. (24) since $S^{(0)} \propto 1 / d^{2}$ in the quasistatic regime. For Au $S^{(0)}$ saturates for small distances so that the quasistatic expression in Eq. (24) is not valid in that case.

To summarize, for distances $d \ll \min \left\{a_{1}, a_{2}\right\}$ the PA gives a good approximation of the impact of surface roughness for all the materials considered here. This allows for estimating the effect of surface roughness from the results obtained for a flat surface and is therefore, an important result of this work. Note, that the same range of validity for the PA has also been reported for the Casimir force. ${ }^{23,36,37}$ In this case, it has also been demonstrated that the theoretical predictions using the PA are in good agreement with the experimental data. $^{38,39}$

\section{CONCLUSION}

We have presented a perturbation theory for determining the surface roughness correction to the heat flux between two semi-infinite bodies. In particular, we have given the explicit expression for the mean Poynting vector up to second-order perturbation theory assuming a Gaussian surface roughness. These results can be employed to estimate the influence of roughness in recent experiments ${ }^{1-4}$ and to study the impact of roughness in thermophotovoltaic devices. ${ }^{8-12}$

In addition, we have discussed the numerical results for two SiC slabs with a given surface roughness. We found that the heat flux becomes larger when surface roughness is taken into account. By giving a detailed discussion of the correction to the transmission coefficients and the spectral Poyn- 
ting vector for two $\mathrm{SiC}$ slabs, we could show that the scattering of surface modes within the rough surfaces causes a larger heat flux which is in contrast to the case of only one rough surface as discussed in Ref. 16.

Finally, we have shown that the PA is valid for distances smaller than the correlation length of the surface roughness regardless of the materials $(\mathrm{SiC}, \mathrm{Au})$ considered in this work. In particular, for the case of two $\mathrm{SiC}$ slabs the validity of the PA is due to an interplay of the specular and diffuse contribution to the mean Poynting vector, whereas for Au which do not have a surface polariton resonance in the infrared region the specular part already gives the PA result. Hence, the PA is valid even when the heat flux is mainly due to the surface polariton coupling giving a simple and therefore, powerful way of estimating the impact of surface roughness to the heat flux.

\section{ACKNOWLEDGMENT}

S.-A.B. gratefully acknowledges support from the Deutsche Akademie der Naturforscher Leopoldina (Grant No. LPDS 2009-7).
${ }^{1}$ L. Hu, A. Narayanaswamy, X. Chen, and G. Chen, Appl. Phys. Lett. 92, 133106 (2008).

${ }^{2}$ A. Narayanaswamy, S. Shen, and G. Chen, Phys. Rev. B 78, 115303 (2008).

${ }^{3}$ S. Shen, A. Narayanaswamy, and G. Chen, Nano Lett. 9, 2909 (2009).

${ }^{4}$ E. Rousseau, A. Siria, G. Jourdan, S. Volz, F. Comin, J. Chevrier, and J.-J. Greffet, Nat. Photonics 3, 514 (2009).

${ }^{5}$ K. Joulain, J.-P. Mulet, F. Marquier, R. Carminati, and J.-J. Greffet, Surf. Sci. Rep. 57, 59 (2005).

${ }^{6}$ A. I. Volokitin and B. N. J. Persson, Rev. Mod. Phys. 79, 1291 (2007).

${ }^{7}$ D. Polder and M. van Hove, Phys. Rev. B 4, 3303 (1971).

${ }^{8}$ R. S. DiMatteo, P. Greiff, S. L. Finberg, K. A. Young-Waithe, H. K. Choy, M. M. Masaki, and C. G. Fonstad, Appl. Phys. Lett. 79, 1894 (2001).

${ }^{9}$ A. Narayanaswamy and G. Chen, Appl. Phys. Lett. 82, 3544 (2003).

${ }^{10}$ M. Laroche, R. Carminati, and J.-J. Greffet, J. Appl. Phys. 100, 063704 (2006).

${ }^{11}$ M. Francoeur, M. P. Mengüç, and R. Vaillon, Appl. Phys. Lett. 93, 043109 (2008).

${ }^{12}$ S. Basu, Z. M. Zhang, and C. J. Fu, Int. J. Energy Res. 33, 1203 (2009).

${ }^{13}$ B. N. J. Persson, B. Lorenz, and A. I. Volokitin, Eur. Phys. J. E 31, 3 (2010).

${ }^{14}$ B. V. Derjaguin, Kolloid-Z. 69, 155 (1934).

${ }^{15}$ J. Błocki, J. Randrup, W. J. Świątecki, and C. F. Tsang, Ann. Phys. 105, 427 (1977).

${ }^{16}$ S.-A. Biehs and J.-J. Greffet, Phys. Rev. B 81, 245414 (2010).

${ }^{17}$ P.-O. Chapuis, S. Volz, C. Henkel, K. Joulain, and J.-J. Greffet, Phys. Rev. B 77, 035431 (2008).

${ }^{18}$ C. Henkel and K. Joulain, Appl. Phys. B: Lasers Opt. 84, 61 (2006).

${ }^{19}$ P. Beckmann and A. Spizzichino, The Scattering of Electromagnetic Waves from Rough Surfaces (Pergamon Press, New York,
1963).

${ }^{20}$ S.-A. Biehs, E. Rousseau, and J.-J. Greffet, Phys. Rev. Lett. 105, 234301 (2010).

${ }^{21}$ J.-J. Greffet, Phys. Rev. B 37, 6436 (1988).

${ }^{22}$ See supplementary material at http://link.aps.org/supplemental/ 10.1103/PhysRevB.82.245410 for a brief discussion of the perturbation theory.

${ }^{23}$ P. A. Maia Neto, A. Lambrecht, and S. Reynaud, Phys. Rev. A 72, 012115 (2005).

${ }^{24}$ A. Lambrecht, P. A. Maia Neto, and S. Reynaud, New J. Phys. 8, 243 (2006).

${ }^{25}$ C. Henkel and V. Sandoghdar, Opt. Commun. 158, 250 (1998).

${ }^{26}$ J. B. Pendry, J. Phys.: Condens. Matter 11, 6621 (1999).

${ }^{27}$ A. V. Shchegrov, K. Joulain, R. Carminati, and J.-J. Greffet, Phys. Rev. Lett. 85, 1548 (2000).

${ }^{28}$ M. L. Levin, V. G. Polevoi, and S. M. Rytov, Zh. Eksp. Teor. Fiz. 79, 2087 (1980) [Sov. Phys. JETP 52, 1054 (1980)].

${ }^{29}$ J. J. Loomis and H. J. Maris, Phys. Rev. B 50, 18517 (1994).

${ }^{30}$ H. J. Simon and J. K. Guha, Opt. Commun. 18, 391 (1976).

${ }^{31}$ H. Raether, Surface Plasmons on Smooth and Rough Surfaces and on Gratings (Springer, Berlin, 1988).

${ }^{32}$ E. N. Economou, Phys. Rev. 182, 539 (1969).

${ }^{33}$ R. Carminati and J.-J. Greffet, Phys. Rev. Lett. 82, 1660 (1999).

${ }^{34}$ C. Henkel, K. Joulain, R. Carminati, and J.-J. Greffet, Opt. Commun. 186, 57 (2000).

${ }^{35}$ W. T. Lau, J.-T. Shen, G. Veronis, and S. Fan, Phys. Rev. E 76, 016601 (2007).

${ }^{36}$ C. Genet, A. Lambrecht, P. A. Maia Neto, and S. Reynaud, Europhys. Lett. 62, 484 (2003).

${ }^{37}$ P. A. M. Neto, A. Lambrecht, and S. Reynaud, Europhys. Lett. 69, 924 (2005).

${ }^{38}$ H. B. Chan, V. A. Aksyuk, R. N. Kleiman, D. J. Bishop, and F. Capasso, Science 291, 1941 (2001).

${ }^{39}$ G. L. Klimchitskaya, U. Mohideen, and V. M. Mostepanenko, Rev. Mod. Phys. 81, 1827 (2009). 